\title{
Noncoherent Spatial/Spectral Optical CDMA System With Two-Dimensional Perfect Difference Codes
}

\author{
Cheing-Hong Lin, Jingshown Wu, Fellow, IEEE, and Chun-Liang Yang
}

\begin{abstract}
In this paper, the family of newly constructed codes, named 2-D perfect difference codes, is proposed to suppress the phase-induced intensity noise (PIIN) in noncoherent spatial/spectral optical code division multiple access (OCDMA) systems. A novel spatial/spectral transceiver structure employing the new codes to eliminate the multiuser interference (MUI) by using the MUI cancellation property of the new codes is also presented. Compared with the systems employing modified quadratic congruence codes (MQC codes) and maximal-area matrices codes (M-matrices codes), numerical results verify that our proposed system can more effectively suppress the PIIN and eliminate MUI. Hence, the number of simultaneous users and total transmission rate increase significantly.
\end{abstract}

Index Terms-Maximal-area matrices codes, modified quadratic congruence codes, multiuser interference, OCDMA, perfect difference codes, phase-induced intensity noise.

\section{INTRODUCTION}

$\mathbf{O}$ PTICAL CODE division multiple access (OCDMA) systems have been investigated for about two decades and received a lot of attention. They have the advantage of providing multiple users to simultaneously access the same bandwidth with high-level security. The conventional OCDMA systems mainly have three kinds of schemes, namely 1) time-spreading scheme [1]-[5]; 2) frequency-hopping scheme [6], [7]; and 3) spectral amplitude-coding (SAC) scheme [8]-[12].

In general, the performance of OCDMA systems is primarily affected by the interference from other simultaneous users, called multiuser interference (MUI) or multiple access interference (MAI). This kind of interference is unavoidable for asynchronous time-spreading systems and frequency-hopping systems. In order to eliminate MUI, Weng et al. [3] proposed the perfect difference codes and a corresponding system. However, the perfect difference codes can only be applied for the synchronous OCDMA system.

In recent years, the SAC-OCDMA systems attract more attention, because MUI can be completely eliminated by spectral coding. Code sequences with fixed in-phase cross correlation such as Hadamard codes have been used to eliminate MUI [8], [9]. However, since the value of the in-phase cross cor-

Manuscript received November 24, 2004; revised June 25, 2005. This work was supported in part by the National Science Council and the Ministry of Education, Taiwan, R.O.C., under Grant NSC 91-2213-E-002-106 and Grant 89E-FA06-2-4-7.

C.-H. Lin and J. Wu are with the Department of Electrical Engineering and Graduate Institute of Communication Engineering, National Taiwan University, Taipei, Taiwan 10617, R.O.C.

C.-L. Yang is with the Department of Electrical Engineering, Tamkang University, Taipei, Taiwan 10617, R.O.C.

Digital Object Identifier 10.1109/JLT.2005.859407 relation between code sequences is large, the phase-induced intensity noise (PIIN) caused by noncoherent broadband light sources of the SAC-OCDMA systems severely degrades the system performance.

In view of this disadvantage, Zhou et al. [10] and Wei et al. [11] used codes with a fixed in-phase cross correlation exactly equal to 1 for suppressing the effect of PIIN. However, since the codes proposed in [10] and [11] are confined in a unidimension, the length of these codes should be made large enough to increase the number of simultaneous users.

In [12], Yang et al. proposed two-dimensional (2-D) maximal-area matrices (M-matrices) codes and a corresponding transceiver structure to shorten the spectral code length and increase the number of simultaneous users. Because the M-matrices codes are derived from one-dimensional (1-D) maximal-length sequence (M-sequence) codes, the cross correlation of the M-matrices codes, similar to that of the Hadamard codes, is very large. It greatly limits the performance of this system.

In this paper, we propose the family of newly constructed codes, named 2-D perfect difference codes. The new codes are constructed in view of the 1-D perfect difference codes [3], which are extended from the codes proposed in [10]. In addition, we also present a corresponding transceiver structure to eliminate the MUI by using an MUI cancellation property of the 2-D perfect difference codes and improve the system performance by suppressing the PIIN.

The rest of this paper is organized as follows. The family of newly constructed codes is described in Section II. Section III presents the proposed system architecture in details. The analytic results of system performance are presented in Section IV. The numerical and simulation results are shown in Section V, and a few discussions are given in Section VI. Finally, we have the conclusion in Section VII.

\section{2-D PERfECt Difference Codes}

As disclosed in [3], the 1-D perfect difference codes are derived from the perfect difference sets, which are defined as follows.

Definition: Let $W_{p}$ be the $v$-set of the integers $0,1, \ldots$, $v-1$ modulo $v$. A set $D=\left\{d_{1}, d_{2}, \ldots, d_{k}\right\}$ is a $k$-subset of $W_{p}$. For every $a \neq 0(\bmod v)$, there are exactly $\lambda$ ordered pairs $\left(d_{i}, d_{j}\right), i \neq j$, such that

$$
d_{i}-d_{j} \equiv a(\bmod v) .
$$

A set $D$ fulfilling these requirements is called a perfect difference set or $(v, k, \lambda)$-cyclic difference set modulo $v$. In 
TABLE I

EXAmples of the Perfect Difference Sets

\begin{tabular}{|c|c|c|c|c|c|c|c|c|c|c|c|c|c|}
\hline$k$ & $v$ & \multicolumn{12}{|c|}{ Perfect difference set } \\
\hline 2 & 3 & 0 & 1 & & & & & & & & & & \\
\hline 3 & 7 & 0 & 1 & 3 & & & & & & & & & \\
\hline 4 & 13 & 0 & 1 & 3 & 9 & & & & & & & & \\
\hline 5 & 21 & 0 & 1 & 4 & 14 & 16 & & & & & & & \\
\hline 6 & 31 & 0 & 1 & 3 & 8 & 12 & 18 & & & & & & \\
\hline 8 & 57 & 0 & 1 & 3 & 13 & 32 & 36 & 43 & 52 & & & & \\
\hline 9 & 73 & 0 & 1 & 3 & 7 & 15 & 31 & 36 & 54 & 63 & & & \\
\hline 10 & 91 & 0 & 1 & 3 & 9 & 27 & 49 & 56 & 61 & 77 & 81 & & \\
\hline 12 & 133 & 0 & 1 & 3 & 12 & 20 & 34 & 38 & 81 & 88 & 94 & 104109 & \\
\hline 14 & 183 & 0 & 1 & 3 & 16 & 23 & 28 & 42 & 76 & 82 & 86 & $\begin{array}{ll}119 & 137\end{array}$ & $154 \quad 175$ \\
\hline
\end{tabular}

accordance with this definition, the $v$ cyclic-shifted sets $D_{\tau}=\left\{d_{1}-\tau, d_{2}-\tau, \ldots, d_{k}-\tau\right\}$ modulo $v$, in which $\tau=$ $0,1, \ldots,(v-1)$, have the same property, where $D_{0}=D$. Furthermore, since there are $k(k-1)$ pairs and the total number for $a \neq 0(\bmod v)$ is $(v-1)$, the relation among $v, k, \lambda$ is

$$
\lambda=\frac{k(k-1)}{v-1} .
$$

In [13], Singer proves the existence of the $(v, k=q+1$, $\lambda=1$ )-cyclic difference set, where $q$ is any power of any prime number (hereinafter, such set is called Singer's perfect difference set for brevity). Some examples of Singer's perfect difference set are shown in Table I. Because Singer's perfect difference set has minimum overlay, i.e., $\lambda=1$, between any two of its cyclic-shifted sets, it is used to construct the 1-D perfect difference codes with the fixed in-phase cross correlation equal to 1 .

The perfect difference codes $C_{\tau}=\left\{c_{\tau, 0}, c_{\tau, 1}, \ldots, c_{\tau, i}\right.$, $\left.c_{\tau,(v-1)}\right\}, \tau \in W_{p}$, are constructed based on Singer's perfect difference set with the rule given as

$$
c_{\tau, i}= \begin{cases}1, & \text { for } i \in D_{\tau} \\ 0, & \text { otherwise. }\end{cases}
$$

The code weight, code length, and code size are $k, v$, and $v$, respectively, where $v=k^{2}-k+1$. An example for $k=5$ is shown in Table II.

The 2-D perfect difference codes in this paper are extended from the 1-D perfect difference codes and can be formed by using two code sequences of the 1-D perfect difference codes. Let $\mathbf{X}=\left[x_{0}, x_{1}, \ldots, x_{M-1}\right]$ and $\mathbf{Y}=\left[y_{0}, y_{1}, \ldots, y_{N-1}\right]$ be two code sequences of the 1-D perfect difference codes. Therein, $M=k_{1}^{2}-k_{1}+1$ and $N=k_{2}^{2}-k_{2}+1$, where $k_{1}$ and $k_{2}$ are the code weights of these two code sequences, respectively, $M$ and $N$ do not need to be the same.

Let $\mathbf{X}_{g}$ and $\mathbf{Y}_{h}$ be the cyclic-shifted versions of $\mathbf{X}$ and $\mathbf{Y}$, respectively, in which $\mathbf{X}_{g}$ is obtained by cyclically shifting $\mathbf{X}$ for $g$ times and $\mathbf{Y}_{h}$ is obtained by cyclically shifting $\mathbf{Y}$ for $h$ times. Then, the 2-D perfect difference codes can be generated by $\mathbf{A}_{g, h}=\mathbf{Y}_{h}^{T} \mathbf{X}_{g}$. An example for $k_{1}=3$ and $k_{2}=2$ is shown in Table III. Accordingly, the code size of the 2-D perfect difference codes is $M \times N$.

In order to explain the cross correlation of the 2-D perfect difference codes, four characteristic matrices $\mathbf{A}^{(d)}, d=0,1,2$, or 3 , are defined as follows according to [12]

$$
\begin{aligned}
& \mathbf{A}^{(0)}=\mathbf{Y}^{T} \mathbf{X} \\
& \mathbf{A}^{(1)}=\mathbf{Y}^{T} \overline{\mathbf{X}} \\
& \mathbf{A}^{(2)}=\overline{\mathbf{Y}}^{T} \mathbf{X}
\end{aligned}
$$

and

$$
\mathbf{A}^{(3)}=\overline{\mathbf{Y}}^{T} \overline{\mathbf{X}}
$$

where $\overline{\mathrm{X}}$ and $\overline{\mathrm{Y}}$ are the complementary code sequences of $\mathrm{X}$ and $\mathbf{Y}$, respectively. The cross correlation between $\mathbf{A}^{(d)}$ and $\mathbf{A}_{g, h}$ can be defined as

$$
R^{(d)}(g, h)=\sum_{i=0}^{M-1} \sum_{j=0}^{N-1} a_{i j}^{(d)} a_{(i+g)(j+h)}
$$

where $a_{i j}^{(d)}$ is the element of the characteristic matrix $\mathbf{A}^{(d)}$ and $a_{(i+g)(j+h)}$ represents the element of $\mathbf{A}_{g, h}$.

In accordance with this definition, the cross correlation of the 2-D perfect difference codes can be expressed as in Table IV. By observing this table, we find that $R^{(3)}(g, h)$ has a nonzero value only when $g \neq 0 \cap h \neq 0$. Furthermore, when $g \neq 0$ and $h \neq 0$, the values of $R^{(0)}(g, h), R^{(1)}(g, h), R^{(2)}(g, h)$, and $R^{(3)}(g, h)$ have a fixed proportion relation.

Therefore, we can use $R^{(3)}(g, h)$ to remove the influence caused by $\mathbf{A}_{g, h}$ with $g \neq 0 \cap h \neq 0$ from $R^{(0)}(g, h)$, $R^{(1)}(g, h)$, and $R^{(2)}(g, h)$ and define three new correlation functions as

$$
R_{I}^{(0)}(g, h)=R^{(0)}(g, h)-\frac{R^{(3)}(g, h)}{\left(k_{1}-1\right)\left(k_{2}-1\right)}
$$


TABLE II

One-Dimensional Perfect DifFERENCE CODES For $k=5$

\begin{tabular}{|c|c|c|c|c|c|c|c|c|c|}
\hline \multirow{2}{*}{$\frac{j}{0}$} & \multicolumn{8}{|c|}{ Cyclic difference sets } & \multirow{2}{*}{$\begin{array}{c}\text { Perfect difference codes } \\
C_{0}=110010000000001010000\end{array}$} \\
\hline & $D_{0}$ & $=\{$ & 0 & 1 & 4 & 14 & 16 & \} & \\
\hline 1 & $D_{1}$ & $=\{2$ & 20 & 0 & 3 & 13 & 15 & \} & $C_{1}=100100000000010100001$ \\
\hline 2 & $D_{2}$ & $=\{$ & 19 & 20 & 2 & 12 & 14 & \} & $C_{2}=001000000000101000011$ \\
\hline 3 & $D_{3}$ & $=\{1$ & 18 & 19 & 1 & 11 & 13 & \} & $C_{3}=010000000001010000110$ \\
\hline 4 & $D_{4}$ & $=\{$ & 17 & 18 & 0 & 10 & 12 & \} & $C_{4}=100000000010100001100$ \\
\hline 5 & $D_{5}$ & $=\{$ & 16 & 17 & 20 & 9 & 11 & \} & 1001 \\
\hline 6 & $D_{6}$ & $=\{$ & 15 & 16 & 19 & 8 & 10 & \} & $C_{6}=000000001010000110010$ \\
\hline 7 & $D_{7}$ & $=\{$ & 14 & 15 & 18 & 7 & 9 & \} & $C_{7}=000000010100001100100$ \\
\hline 8 & $D_{8}$ & $=\{$ & 13 & 14 & 17 & 6 & 8 & \} & 1001000 \\
\hline 9 & $D_{9}$ & $=\{$ & 12 & 13 & 16 & 5 & 7 & \} & $C_{9}=000001010000110010000$ \\
\hline 10 & $D_{10}$ & $=\{$ & 11 & 12 & 15 & 4 & 6 & \} & 100100000 \\
\hline 11 & $D_{11}$ & $=\{$ & 10 & 11 & 14 & 3 & 5 & \} & $C_{11}=000101000011001000000$ \\
\hline 12 & $D_{12}$ & $=\{$ & 9 & 10 & 13 & 2 & 4 & \} & $C_{12}=001010000110010000000$ \\
\hline 13 & $D_{13}$ & $=\{$ & 8 & 9 & 12 & 1 & 3 & \} & $C_{13}=010100001100100000000$ \\
\hline 14 & $D_{14}$ & $=\{$ & 7 & 8 & 11 & 0 & 2 & \} & $C_{14}=101000011001000000000$ \\
\hline 15 & $D_{15}$ & $=\{$ & 6 & 7 & 10 & 20 & 1 & \} & $C_{15}=010000110010000000001$ \\
\hline 16 & $D_{16}$ & $=\{$ & 5 & 6 & 9 & 19 & 0 & \} & $C_{16}=100001100100000000010$ \\
\hline 17 & $D_{17}$ & $=\{$ & 4 & 5 & 8 & 18 & 20 & \} & $C_{17}=000011001000000000101$ \\
\hline 18 & $D_{18}$ & $=\{$ & 3 & 4 & 7 & 17 & 19 & \} & $C_{18}=000110010000000001010$ \\
\hline 19 & $D_{19}$ & $=\{$ & 2 & 3 & 6 & 16 & 18 & \} & $C_{19}=001100100000000010100$ \\
\hline 20 & $D_{20}$ & $=\{$ & 1 & 2 & 5 & 15 & 17 & \} & $C_{20}=011001000000000101000$ \\
\hline
\end{tabular}

TABLE III

Two-Dimensional Perfect Difference Codes for $k_{1}=3$ And $k_{2}=2$

\begin{tabular}{|c|c|c|c|c|c|c|c|c|}
\hline $\mathbf{A}_{g, h}$ & {$[1101000]$} & {$[0110100]$} & {$[0011010]$} & {$[0001101]$} & {$[\mathbf{1 0 0 0 1 1 0}]$} & {$[0100011]$} & {$[1010001]$} & $\mathbf{X}_{g}$ \\
\hline$[1]$ & {$[1101000]$} & {$[0110100]$} & {$[0011010]$} & {$[0001101]$} & {$[1000110]$} & {$[0100011]$} & {$[1010001]$} & \\
\hline 1 & 1101000 & 0110100 & 0011010 & 0001101 & 1000110 & 0100011 & 1010001 & \\
\hline 0 & 0000000 & 0000000 & 0000000 & 0000000 & 0000000 & 0000000 & 0000000 & \\
\hline$[0$ & {$[0000000]$} & 0000000 & {$[0000000]$} & {$[0000000]$} & {$[0000000]$} & $0000000]$ & {$[0000000]$} & \\
\hline 1 & 1101000 & 0110100 & 0011010 & 0001101 & 1000110 & 0100011 & 1010001 & \\
\hline 1 & 1101000 & 0110100 & 0011010 & 0001101 & 1000110 & 0100011 & 1010001 & \\
\hline$[1]$ & {$[1101000]$} & 0110100 & {$[0011010]$} & {$[0001101]$} & {$[1000110]$} & 0100011 & {$[1010001]$} & \\
\hline $\mathbf{0}$ & 0000000 & 0000000 & 0000000 & 0000000 & 0000000 & 0000000 & 0000000 & \\
\hline 1 & 1101000 & 0110100 & 0011010 & 0001101 & 1000110 & 0100011 & 1010001 & \\
\hline $\mathbf{Y}_{h}$ & & & & & & & & \\
\hline
\end{tabular}


TABLE IV

Cross-Correlations of the 2-D Perfect Difference Codes

\begin{tabular}{|l|c|c|c|c|}
\hline correlation & $R^{(0)}(g, h)$ & $R^{(1)}(g, h)$ & $R^{(2)}(g, h)$ & $R^{(3)}(g, h)$ \\
\hline $\mathrm{A}_{g, h}=0, h=0$ & $k_{1} \cdot k_{2}$ & 0 & 0 & 0 \\
\hline$g=0, h \neq 0$ & $k_{1}$ & 0 & $k_{1} \cdot\left(k_{2}-1\right)$ & 0 \\
\hline$g \neq 0, h=0$ & $k_{2}$ & $\left(k_{1}-1\right) \cdot k_{2}$ & 0 & 0 \\
\hline$g \neq 0, h \neq 0$ & 1 & $\left(k_{1}-1\right)$ & $\left(k_{2}-1\right)$ & $\left(k_{1}-1\right) \cdot\left(k_{2}-1\right)$ \\
\hline
\end{tabular}

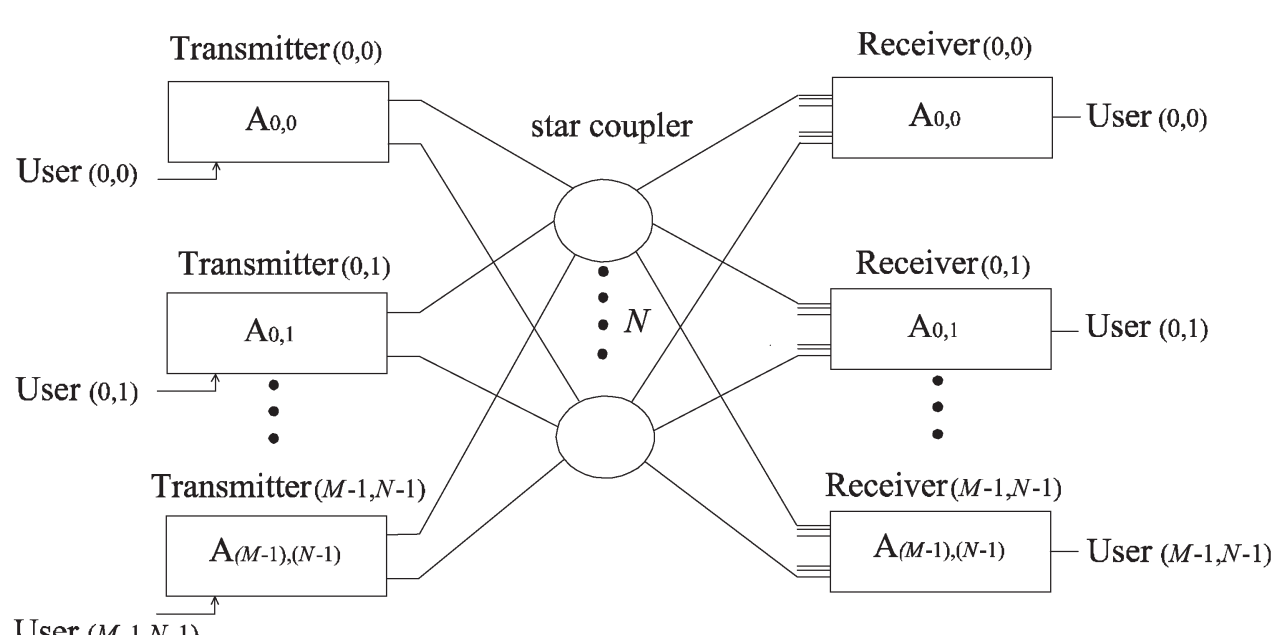

Fig. 1. Schematic of a spectral/spatial OCDMA network.

$$
R_{I}^{(1)}(g, h)=R^{(1)}(g, h)-\frac{R^{(3)}(g, h)}{\left(k_{2}-1\right)}
$$

and

$$
R_{I}^{(2)}(g, h)=R^{(2)}(g, h)-\frac{R^{(3)}(g, h)}{\left(k_{1}-1\right)} .
$$

Likewise, $R_{I}^{(1)}(g, h)$ and $R_{I}^{(2)}(g, h)$ have nonzero values only when $g \neq 0 \cap h=0$ and $g=0 \cap h \neq 0$, respectively. Hence, they can be used to remove the influence caused by $\mathbf{A}_{g, h}$ with $g \neq 0 \cap h=0$ or $g=0 \cap h \neq 0$ from $R_{I}^{(0)}(g, h)$. Thus, we can find the following property:

$$
\begin{aligned}
R_{I}^{(0)}(g, h)-\frac{R_{I}^{(1)}(g, h)}{\left(k_{1}-1\right)} & -\frac{R_{I}^{(2)}(g, h)}{\left(k_{2}-1\right)} \\
= & \begin{cases}k_{1} k_{2}, & \text { for } g=0 \text { and } h=0 \\
0, & \text { otherwise. }\end{cases}
\end{aligned}
$$

Substituting (9)-(11) into (12), we can obtain an important property given as

$$
\begin{aligned}
& R^{(0)}(g, h)-\frac{1}{k_{1}-1} R^{(1)}(g, h)-\frac{1}{k_{2}-1} R^{(2)}(g, h) \\
& +\frac{1}{\left(k_{1}-1\right)\left(k_{2}-1\right)} R^{(3)}(g, h) \\
& \quad= \begin{cases}k_{1} k_{2}, & \text { for } g=0 \text { and } h=0 \\
0, & \text { otherwise. }\end{cases}
\end{aligned}
$$

This property is used to cancel the MUI and suppress the PIIN in the following section.

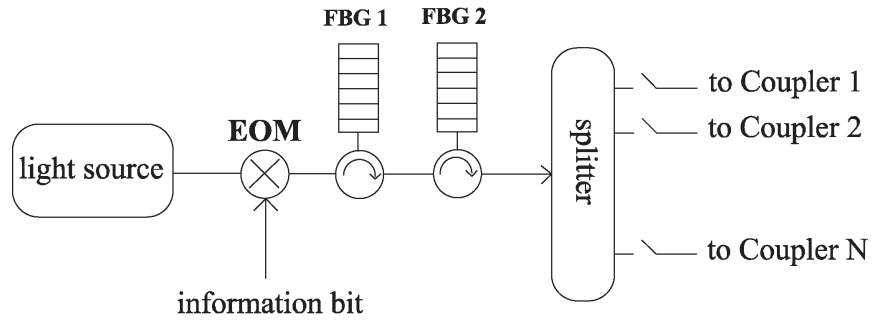

Fig. 2. Transmitter structure of the proposed spectral/spatial OCDMA system.

\section{SYSTEM DESCRIPTION}

Fig. 1 shows the spatial/spectral OCDMA network, which includes $M N$ transmitters/receiver pairs and $N$ star couplers. Every transmitter/receiver pair is assigned with a 2-D perfect difference codeword $\mathbf{A}_{g, h}, g=0,1,2, \ldots, M-1$ and $h=0$, $1,2, \ldots, N-1$. The transmitter first encodes the incoming binary data with their corresponding codewords and then broadcast the encoded data sequences to the receivers via the star couplers. After the encoded data sequences are received, the receiver uses double-balanced detectors to abstract the desired information bits according to the corresponding codewords [12].

Fig. 2 shows the structure of the transmitter, which is composed of an unpolarized broadband light source, an electrooptic modulator (EOM), two sets of fiber Bragg gratings (FBGs), i.e., FBG1 and FBG2, and an optical splitter. Therein, the broadband light source has a flat spectrum. Moreover, FBGs 1 and 2 have the same number of gratings and can be used to reflect back the same spectral components.

Initially, the EOM modulates the incoming information bits by using the ON-OFF keying scheme. Thereby, the electronic 
data are converted to broadband optical pulses. Then, these optical pulses are delivered to the two sets of FBGs and the splitter for encoding. Since each 2-D perfect difference codeword $\mathbf{A}_{g, h}$ is generated by using two specific 1-D perfect difference code sequences, i.e., $\mathbf{X}_{g}$ and $\mathbf{Y}_{h}$, we take one of them for spectral encoding and the other one for spatial encoding. (Thereinafter, these two code sequences are called spectral and spatial code sequences, respectively.) The spectral encoding and spatial encoding are performed by using the two sets of FBGs and splitter, respectively.

When an optical pulse is inputted into FBG1, the spectral components matched to " $1 \mathrm{~s}$ " of the spectral code sequence are reflected back, and the others are filtered out. However, the matched spectral components are reflected by different gratings. It causes the round-trip delays of the matched spectral components to be different. In order to compensate the different round-trip delays of the matched spectral components, we make the arrangement of the gratings in FBG2 contrary to that in FBG1 so that all the matched spectral components have the same delay time and are sent at the same moment.

After spectral encoding, the optical pulses are passed to the splitter for spatial encoding. The splitter equally splits each optical pulse and sends these split portions to the star couplers according to the spatial code sequence. For example, if the code weight of the spatial code sequence is $k_{s}$, the splitter will equally divide the power of the optical pulse into $k_{s}$ portions and, respectively, send these portions to the star couplers matched to " $1 \mathrm{~s}$ " of the spatial code sequence.

Fig. 3 shows the structure of the receivers. Each of the receivers is composed of two optical combiners and two balanced detectors. Every balanced detector has two sets of FBGs and two p-i-n photodiodes. Therein, FBGs $1-4$ of the balanced detectors have the same number of gratings and can be used to reflect back the same spectral components. Again, in order to compensate the different round-trip delays of matched spectral components, the arrangements of the gratings in FBGs 1 and 3 are the same but contrary to that in FBGs 2 and 4.

The receiver structure shown in Fig. 3 is constructed to realize the MUI cancellation property (13) and recover the information bits thereby. The optical combiner in the upper branch only receives and combines the signals sent from the star couplers matched to " $1 \mathrm{~s}$ " of the spatial code sequence so that the interference caused by the transmitters with different spatial code sequences can be mostly removed. Then, the received signals are delivered to FBG1 of the upper balanced detector.

Similar to the transmitter, FBG1 of the upper balanced detector can reflect back the spectral components matched to " $1 \mathrm{~s}$ " of the spectral code sequence and filter out the others. Hence, the spectral components of the received signals are divided into two portions. The matched portion is passed to FBG2 of the upper balanced detector for delay compensation and then passed to PD0. The unmatched portion is passed to PD1 via a delay line and a $1 /\left(k_{1}-1\right)$ optical attenuator. Therein, the delay line is used to compensate the delay caused by FBGs 1 and 2 .

The optical combiner in the lower branch only receives and combines the signals sent from the star couplers matched to "Os" of the spatial code sequence to obtain the interference information. After being received by the lower combiner, the signals are passed to the FBG3, which can reflect back the spectral components matched to " $1 \mathrm{~s}$ " of the spectral code sequence so as to divide the signals into two portions.

The reflected portion is passed to FBG4 for delay compensation and then passed to PD2 via a $1 /\left(k_{2}-1\right)$ optical attenuator. The other portion is passed to PD3 via a delay line and a $1 /\left(k_{1}-1\right)\left(k_{2}-1\right)$ optical attenuator. Therein, the delay line is used to compensate the delay caused by FBGs 3 and 4.

Since only the signal components matched to the spatial and spectral code sequences of the receiver can reach PD0, the output current of PD0 is proportional to $R^{(0)}(g, h)$. Likewise, the currents outputted from PDs 1-3 are proportional to $R^{(1)}(g, h) /\left(k_{1}-1\right), \quad R^{(2)}(g, h) /\left(k_{2}-1\right)$ and $R^{(3)}(g, h) /$ $\left(k_{1}-1\right)\left(k_{2}-1\right)$, respectively. Thus, the output currents of the upper and lower balanced detectors are proportional to $\left[R^{(0)}(g, h)-R^{(1)}(g, h) /\left(k_{1}-1\right)\right]$ and $\left[R^{(2)}(g, h) /\left(k_{2}-1\right)-\right.$ $\left.R^{(3)}(g, h) /\left(k_{1}-1\right)\left(k_{2}-1\right)\right]$, respectively. Finally, the output current of the receiver is proportional to $\left[R^{(0)}(g, h)-\right.$ $R^{(1)}(g, h) /\left(k_{1}-1\right)-R^{(2)}(g, h) /\left(k_{2}-1\right)+R^{(3)}(g, h) /\left(k_{1}-1\right)$ $\left.\left(k_{2}-1\right)\right]$.

Therefore, according to the MUI cancellation property (13), the receiver can completely eliminate the interference from the undesired transmitters using unmatched codewords and recover the desired information bits. Moreover, since three optical attenuators are used in the receiver to lower the interference power inputted to the photodiodes, the PIIN can be suppressed further.

\section{Performance Analysis}

We consider the coherent intensity noise, as well as shot and thermal noises in the photodiodes. The effect of the receiver's dark current is neglected. Gaussian approximation is employed to calculate the bit error rate (BER). The general form of the photocurrent noise caused by using a photodiode to detect a thermal light (emitted by a broadband light source) can be expressed as [11], [14], [15]

$$
\left\langle i^{2}\right\rangle=I^{2} B \tau_{c}+2 e I B+\frac{4 K_{b} T_{n} B}{R_{L}}
$$

where $I$ is the average photocurrent output from the photodiode, $B$ is the electrical bandwidth, $\tau_{c}$ is the coherence time of the light beam inputted to the photodiode, $e$ is electron's charge, $K_{b}$ is Boltzmann's constant, $T_{n}$ is the absolute noise temperature, and $R_{L}$ is the load resistance. Therein, $\tau_{c}$ can be expressed as [14]

$$
\tau_{c}=\frac{\int_{0}^{\infty} S^{2}(f) d f}{\left[\int_{0}^{\infty} S(f) d f\right]^{2}}
$$

where $S(f)$ is the single sideband power spectral density (PSD) of the light incident to the photodiode. Moreover, items at the right-hand side of (14) are results of the PIIN, shot noise, and thermal noise, respectively. 


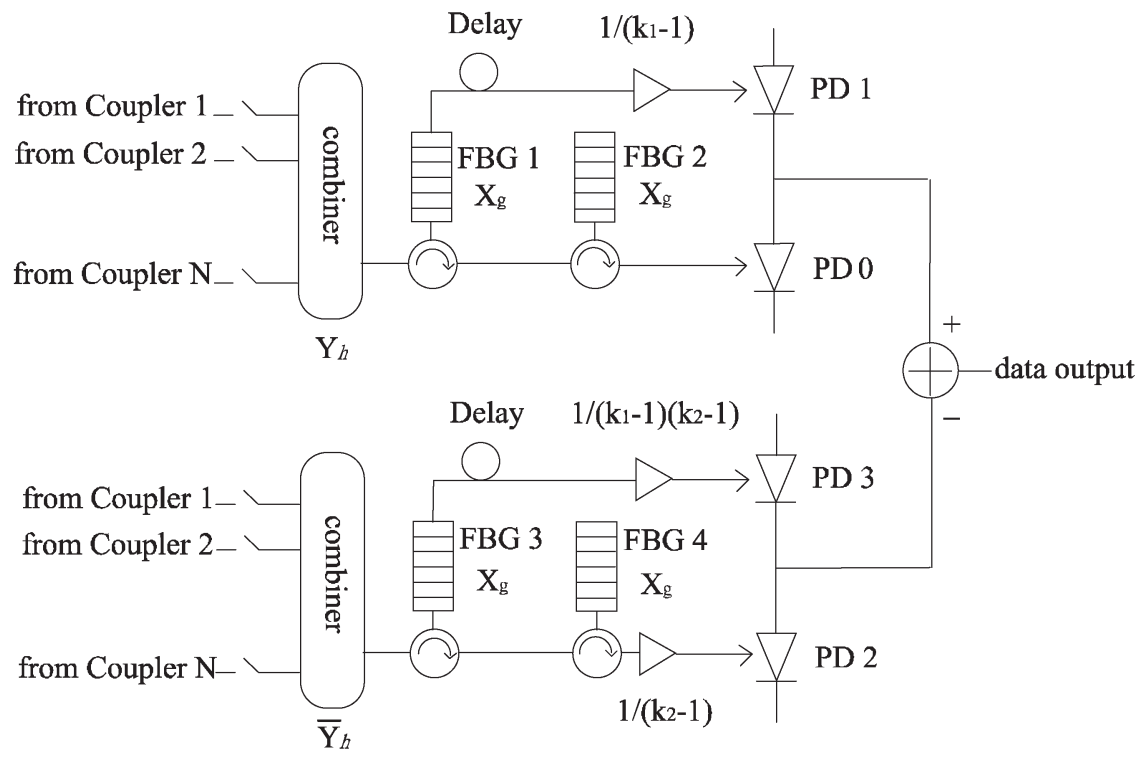

Fig. 3. Receiver structure of the proposed spectral/spatial OCDMA system.

Furthermore, in order to simplify the analysis, we make some assumptions as follows. First, the broadband light source is ideally unpolarized and has flat spectrum over $\left[f_{o}-\Delta f / 2, f_{o}+\right.$ $\Delta f / 2]$, where $f_{o}$ and $\Delta f$ are the central frequency and the bandwidth of the source. Second, each of the spectral components has identical spectral width. Third, each user has equal power at the receivers. Last, bit streams sent from different transmitters are synchronously arrived at the receivers.

It should be noted that the last assumption is made just for convenience of performance analysis. In fact, the bit streams from different transmitters may asynchronously arrive at the receivers. Since the proposed system can completely remove MUI automatically without the synchronization requirement, the system performance will not be affected by MUI even though the bit streams from undesired transmitters arrived asynchronously. As for PIIN, because the ensemble effects caused in the synchronous and asynchronous cases are the same, the system performance must be the same as these two cases.

Based on the assumptions above, the PSD of the received optical signals can be written as

$$
\begin{aligned}
& r(f)= \frac{P_{\mathrm{sr}}}{k_{2} \Delta f} \sum_{w=1}^{W} d(w) \cdot \sum_{i=0}^{M-1} \sum_{j=0}^{N-1} a_{i j}(w) \\
& \times\left\{u\left[f-f_{o}-\frac{\Delta f}{2 M}(-M+2 i)\right]\right. \\
&\left.-u\left[f-f_{o}-\frac{\Delta f}{2 M}(-M+2 i+2)\right]\right\}
\end{aligned}
$$

where $P_{\mathrm{sr}}$ is the effective source power at the receiver, $\Delta f$ is the bandwidth of the source, $k_{2}$ is the code weight of the spatial code sequence, $W$ is the number of active users, $d(w)$ is the data bit of the $w$ th user, which can be " 1 " or " 0 ," $M$ and $N$ are the code lengths of the spectral and spatial code sequences, respectively, $a_{i j}(w)$ represents an element of the $w$ th user's codeword, and $u(f)$ is the unit step function defined as

$$
u(f)= \begin{cases}1, & f \geq 0 \\ 0, & f<0 .\end{cases}
$$

Therefore, the PSDs at PDs $0-3$ of the receiver using the codeword $\mathbf{A}_{0,0}$ during one bit period can be written as

$$
\begin{aligned}
G_{0}(f)= & \frac{P_{\mathrm{sr}}}{k_{2} \Delta f} \sum_{w=1}^{W} d(w) \cdot \sum_{i=0}^{M-1} \sum_{j=0}^{N-1} a_{i j}^{(0)} a_{i j}(w) \\
& \times\left\{u\left[f-f_{o}-\frac{\Delta f}{2 M}(-M+2 i)\right]\right. \\
& \left.-u\left[f-f_{o}-\frac{\Delta f}{2 M}(-M+2 i+2)\right]\right\} \\
G_{1}(f)= & \frac{P_{\mathrm{sr}}}{\left(k_{1}-1\right) k_{2} \Delta f} \sum_{w=1}^{W} d(w) \cdot \sum_{i=0}^{M-1} \sum_{j=0}^{N-1} a_{i j}^{(1)} a_{i j}(w) \\
& \times\left\{u\left[f-f_{o}-\frac{\Delta f}{2 M}(-M+2 i)\right]\right. \\
G_{2}(f)= & \frac{P_{\mathrm{sr}}}{\left(k_{2}-1\right) k_{2} \Delta f} \sum_{w=1}^{W} d(w) \cdot \sum_{i=0}^{M-1} \sum_{j=0}^{N-1} a_{i j}^{(2)} a_{i j}(w) \\
& \times\left\{u\left[f-f_{o}-\frac{\Delta f}{2 M}(-M+2 i)\right]\right. \\
& \left.\quad-u\left[f-f_{o}-\frac{\Delta f}{2 M}(-M+2 i+2)\right]\right\}
\end{aligned}
$$


and

$$
\begin{array}{r}
G_{3}(f)=\frac{P_{\mathrm{sr}}}{\left(k_{1}-1\right)\left(k_{2}-1\right) k_{2} \Delta f} \sum_{w=1}^{W} d(w) \cdot \sum_{i=0}^{M-1} \sum_{j=0}^{N-1} a_{i j}^{(3)} a_{i j}(w) \\
\times\left\{u\left[f-f_{o}-\frac{\Delta f}{2 M}(-M+2 i)\right]\right. \\
\left.-u\left[f-f_{o}-\frac{\Delta f}{2 M}(-M+2 i+2)\right]\right\}
\end{array}
$$

where $k_{1}$ is the code weight of the spectral code sequence.

As shown in Table IV, the codewords are divided into four groups, i.e., $\mathbf{A}_{g, h}$ with $g=0 \cap h=0, g \neq 0 \cap h=0, g=0 \cap$ $h \neq 0$, and $g \neq 0 \cap h \neq 0$. The numbers of these groups are 1 , $(M-1),(N-1)$, and $(M-1)(N-1)$, respectively. Thus, the active users can also be divided into four groups according to their codewords. For convenience, we use the ensemble means of the amounts of these four groups for calculation, i.e., $1,(W-1)(M-1) /(M N-1),(W-1)(N-1) /(M N-1)$, and $(W-1)(M-1)(N-1) /(M N-1)$, respectively. To simplify the calculation, we first let $d(w)=1$ and $w=1,2$, $\ldots, W$. The average output currents of PDs $0-3$ of the desired receiver can be expressed as

$$
\begin{aligned}
& I_{0}=R \int_{0}^{\infty} G_{0}(f) d f \\
&=\frac{R P_{\mathrm{sr}}}{M k_{2}}\left\{k_{1} k_{2}+\frac{(W-1)(N-1)}{(M N-1)} k_{1}+\frac{(W-1)(M-1)}{(M N-1)} k_{2}\right. \\
& \qquad \\
& \qquad \\
&\left.\qquad \frac{(W-1)(M-1)(N-1)}{(M N-1)}\right\}
\end{aligned}
$$

$$
\begin{aligned}
I_{1}=R \int_{0}^{\infty} G_{1}(f) d f & \\
= & \frac{R P_{\mathrm{sr}}}{M k_{2}\left(k_{1}-1\right)}\left\{\frac{(W-1)(M-1)}{(M N-1)} k_{2}\left(k_{1}-1\right)\right. \\
& \left.\quad+\frac{(W-1)(M-1)(N-1)}{(M N-1)}\left(k_{1}-1\right)\right\} \\
= & \frac{R P_{\mathrm{sr}}}{M k_{2}}\left\{\frac{(W-1)(M-1)}{(M N-1)} k_{2}+\frac{(W-1)(M-1)(N-1)}{(M N-1)}\right\}
\end{aligned}
$$

$$
\begin{aligned}
I_{2}=R \int_{0}^{\infty} G_{2}(f) d f & \\
= & \frac{R P_{\mathrm{sr}}}{M k_{2}\left(k_{2}-1\right)}\left\{\frac{(W-1)(N-1)}{(M N-1)} k_{1}\left(k_{2}-1\right)\right. \\
& \left.\quad+\frac{(W-1)(M-1)(N-1)}{(M N-1)}\left(k_{2}-1\right)\right\} \\
= & \frac{R P_{\mathrm{sr}}}{M k_{2}}\left\{\frac{(W-1)(N-1)}{(M N-1)} k_{1}+\frac{(W-1)(M-1)(N-1)}{(M N-1)}\right\}
\end{aligned}
$$

and

$$
\begin{aligned}
I_{3}= & R \int_{0}^{\infty} G_{3}(f) d f \\
= & \frac{R P_{\mathrm{sr}}}{M k_{2}\left(k_{1}-1\right)\left(k_{2}-1\right)} \\
& \cdot\left\{\frac{(W-1)(M-1)(N-1)}{(M N-1)}\left(k_{1}-1\right)\left(k_{2}-1\right)\right\} \\
= & \frac{R P_{\mathrm{sr}}}{M k_{2}}\left\{\frac{(W-1)(M-1)(N-1)}{(M N-1)}\right\}
\end{aligned}
$$

where $R$ is the responsivity of the photodiode given by $R=$ $\eta e / h f_{o}, \eta$ is the quantum efficiency of the photodiode, $h$ is Planck's constant, and $f_{o}$ is the central frequency of the incident light. Consequently, the total photocurrent output from the receiver can be written as

$$
\begin{aligned}
I_{\mathrm{r}} & =R \int_{0}^{\infty}\left[G_{0}(f)-G_{1}(f)-G_{2}(f)+G_{3}(f)\right] d f \\
& =\left(I_{0}-I_{1}\right)-\left(I_{2}-I_{3}\right) \\
& =\frac{R P_{\mathrm{sr}} k_{1}}{M} .
\end{aligned}
$$

The power of PIIN that exists in the photocurrent of the receiver can be written as [11], [16]

$$
\begin{aligned}
\left\langle i_{\mathrm{PIIN}}^{2}\right\rangle & =B_{\mathrm{r}} I_{\mathrm{r}}^{2} \tau_{\mathrm{r}} \\
& =B_{\mathrm{r}} I_{\mathrm{r}}^{2} \frac{\int_{0}^{\infty}\left[G_{0}(f)-G_{1}(f)-G_{2}(f)+G_{3}(f)\right]^{2} d f}{\left\{\int_{0}^{\infty}\left[G_{0}(f)-G_{1}(f)-G_{2}(f)+G_{3}(f)\right] d f\right\}^{2}} \\
& =B_{\mathrm{r}} R^{2} \int_{0}^{\infty}\left[G_{0}(f)-G_{1}(f)-G_{2}(f)+G_{3}(f)\right]^{2} d f
\end{aligned}
$$

where $\tau_{\mathrm{r}}$ is the coherence time of the optical signals received, and $B_{\mathrm{r}}$ is the electrical bandwidth of the receiver.

Since $G_{0}(f)$ and $G_{2}(f)$ do not overlap the spectrum of $G_{1}(f)$ and $G_{3}(f)$, PIIN can be rewritten as

$$
\begin{array}{r}
\left\langle i_{\mathrm{PIIN}}^{2}\right\rangle=B_{\mathrm{r}} R^{2} \int_{0}^{\infty}\left[G_{0}^{2}(f)+G_{1}^{2}(f)+G_{2}^{2}(f)+G_{3}^{2}(f)\right. \\
\left.-2 G_{0}(f) G_{2}(f)-2 G_{1}(f) G_{3}(f)\right] d f .
\end{array}
$$


According to (18), we have

$$
\begin{aligned}
\int_{0}^{\infty} G_{0}^{2}(f) d f & \\
= & \frac{P_{\mathrm{sr}}^{2}}{k_{2}^{2} \Delta f^{2}} \int_{0}^{\infty}\left[\sum_{w=1}^{W} \sum_{i=0}^{M-1} \sum_{j=0}^{N-1} a_{i j}^{(0)} a_{i j}(w) F(f, i)\right]^{2} d f \\
= & \frac{P_{\mathrm{sr}}^{2}}{k_{2}^{2} \Delta f^{2}} \frac{\Delta f}{M} \sum_{i=0}^{M-1}\left[\sum_{w=1}^{W} \sum_{j=0}^{N-1} a_{i j}^{(0)} a_{i j}(w)\right]^{2} \\
= & \frac{P_{\mathrm{sr}}^{2}}{k_{2}^{2} M \Delta f} \\
& \cdot k_{1}\left[k_{2}+\frac{k_{2}}{k_{1}} \frac{(W-1)(M-1)}{(M N-1)}+\frac{(W-1)(N-1)}{(M N-1)}\right. \\
& \left.+\frac{1}{k_{1}} \frac{(W-1)(M-1)(N-1)}{(M N-1)}\right]^{2} \\
= & \frac{P_{\mathrm{sr}}^{2}}{M \Delta f k_{2}^{2} k_{1}(M N-1)^{2}} \\
& \cdot\left[k_{1} k_{2}(M N-1)+k_{2}(W-1)(M-1)\right. \\
& \left.+k_{1}(W-1)(N-1)+(W-1)(N-1)(M-1)\right]^{2}
\end{aligned}
$$

where

$$
\begin{aligned}
F(f, i)=\{u[f & \left.-f_{o}-\frac{\Delta f}{2 M}(-M+2 i)\right] \\
& \left.-u\left[f-f_{o}-\frac{\Delta f}{2 M}(-M+2 i+2)\right]\right\} .
\end{aligned}
$$

Likewise, according to (19)-(21), we have

$$
\begin{aligned}
\int_{0}^{\infty} G_{1}^{2}(f) d f & \\
= & \frac{P_{\mathrm{sr}}^{2}}{\left(k_{1}-1\right)^{2} k_{2}^{2} \Delta f^{2}} \\
& \cdot \int_{0}^{\infty}\left[\sum_{w=1}^{W} \sum_{i=0}^{M-1} \sum_{j=0}^{N-1} a_{i j}^{(1)} a_{i j}(w) F(f, i)\right]^{2} d f \\
= & \frac{P_{\mathrm{sr}}^{2}}{\left(k_{1}-1\right)^{2} k_{2}^{2} \Delta f^{2}} \frac{\Delta f}{M} \sum_{i=0}^{M-1}\left[\sum_{w=1}^{W} \sum_{j=0}^{N-1} a_{i j}^{(1)} a_{i j}(w)\right]^{2} \\
& \times\left[\frac{P_{\mathrm{sr}}^{2}}{\left(k_{1}-1\right)^{2} k_{2}^{2} M \Delta f} \cdot\left(k_{1}-1\right)^{2}\right. \\
& \left.+\frac{(W-1)(M-1)}{\left(k_{1}-1\right)} \frac{(W-1)(M-1)(N-1)}{(M N-1)}\right]^{2}
\end{aligned}
$$

$$
\begin{aligned}
= & \frac{P_{\mathrm{sr}}^{2}}{M \Delta f k_{2}^{2}\left(k_{1}-1\right)^{2}(M N-1)^{2}} \\
& \cdot\left[k_{2}(W-1)(M-1)+(W-1)(N-1)(M-1)\right]^{2}
\end{aligned}
$$

$$
\begin{aligned}
\int_{0}^{\infty} G_{2}^{2}(f) d f & \\
= & \frac{P_{\mathrm{sr}}^{2}}{\left(k_{2}-1\right)^{2} k_{2}^{2} \Delta f^{2}} \\
= & \int_{0}^{\infty}\left[\sum_{w=1}^{W} \sum_{i=0}^{M-1} \sum_{j=0}^{N-1} a_{i j}^{(2)} a_{i j}(w) F(f, i)\right]^{2} d f \\
= & \frac{P_{\mathrm{sr}}^{2}}{\left(k_{2}-1\right)^{2} k_{2}^{2} \Delta f^{2}} \frac{\Delta f}{M} \sum_{i=0}^{M-1}\left[\sum_{w=1}^{W} \sum_{j=0}^{N-1} a_{i j}^{(2)} a_{i j}(w)\right]^{2} \\
= & \frac{P_{\mathrm{sr}}^{2}}{M \Delta f k_{2}^{2} k_{1}(M N-1)^{2}} \\
& \cdot k_{1}\left[\left(k_{2}-1\right) \frac{(W-1)(N-1)}{(M N-1)}\right. \\
& \left.+\frac{\left(k_{2}-1\right)}{k_{1}} \frac{(W-1)(M-1)(N-1)}{(M N-1)}\right]^{2}
\end{aligned}
$$

$\int_{0}^{\infty} G_{3}^{2}(f) d f$

$$
\begin{aligned}
= & \frac{P_{\mathrm{sr}}^{2}}{\left(k_{1}-1\right)^{2}\left(k_{2}-1\right)^{2} k_{2}^{2} \Delta f^{2}} \\
& \cdot \int_{0}^{\infty}\left[\sum_{w=1}^{W} \sum_{i=0}^{M-1} \sum_{j=0}^{N-1} a_{i j}^{(3)} a_{i j}(w) F(f, i)\right]^{2} d f \\
= & \frac{P_{\mathrm{sr}}^{2}}{\left(k_{1}-1\right)^{2}\left(k_{2}-1\right)^{2} k_{2}^{2} \Delta f^{2}} \\
= & \frac{\Delta f}{M} \sum_{i=0}^{M-1}\left[\sum_{w=1}^{W} \sum_{j=0}^{N-1} a_{i j}^{(3)} a_{i j}(w)\right]^{2} \\
& \cdot\left(k_{1}-1\right)^{2}\left(k_{2}-1\right)^{2} k_{2}^{2} M \Delta f \\
= & \frac{P_{\mathrm{sr}}^{2}}{M \Delta f k_{2}^{2}\left(k_{1}-1\right)^{2}(M N-1)^{2}} \\
& \cdot[(W-1)(N-1)(M-1)]^{2} \\
\left(k_{1}-1\right) & \frac{(W-1)(M-1)(N-1)}{\left(k_{\mathrm{sr}}-1\right)} \\
& \frac{(M N-1)}{(N-1)}
\end{aligned}
$$




$$
\begin{aligned}
& \int_{0}^{\infty} G_{0}(f) G_{2}(f) d f \\
& =\frac{P_{\mathrm{sr}}^{2}}{\left(k_{2}-1\right) k_{2}^{2} \Delta f^{2}} \\
& \cdot \int_{0}^{\infty}\left[\sum_{w_{1}=1}^{W} \sum_{i_{1}=0}^{M-1} \sum_{j_{1}=0}^{N-1} a_{i_{1} j_{1}}^{(0)} a_{i_{1} j_{1}}\left(w_{1}\right) F\left(f, i_{1}\right)\right] \\
& \text {. }\left[\sum_{w_{2}=1}^{W} \sum_{i_{2}=0}^{M-1} \sum_{j_{2}=0}^{N-1} a_{i_{2} j_{2}}^{(2)} a_{i_{2} j_{2}}\left(w_{2}\right) F\left(f, i_{2}\right)\right] d f \\
& =\frac{P_{\mathrm{sr}}^{2}}{\left(k_{2}-1\right) k_{2}^{2} \Delta f^{2}} \\
& \cdot \frac{\Delta f}{M} \sum_{i=0}^{M-1}\left\{\left[\sum_{w_{1}=1}^{W} \sum_{j_{1}=0}^{N-1} a_{i_{1} j_{1}}^{(0)} a_{i_{1} j_{1}}\left(w_{1}\right)\right]\right. \\
& \left.\cdot\left[\sum_{w_{2}=1}^{W} \sum_{j_{2}=0}^{N-1} a_{i_{2} j_{2}}^{(2)} a_{i_{2} j_{2}}\left(w_{2}\right)\right]\right\} \\
& =\frac{P_{\mathrm{sr}}^{2}}{\left(k_{2}-1\right) k_{2}^{2} M \Delta f} \\
& \cdot k_{1}\left[k_{2}+\frac{k_{2}}{k_{1}} \frac{(W-1)(M-1)}{(M N-1)}+\frac{(W-1)(N-1)}{(M N-1)}\right. \\
& \left.+\frac{1}{k_{1}} \frac{(W-1)(M-1)(N-1)}{(M N-1)}\right] \\
& \cdot\left[\left(k_{2}-1\right) \frac{(W-1)(N-1)}{(M N-1)}\right. \\
& \left.+\frac{\left(k_{2}-1\right)}{k_{1}} \frac{(W-1)(M-1)(N-1)}{(M N-1)}\right] \\
& =\frac{P_{\mathrm{sr}}^{2}}{M \Delta f k_{2}^{2} k_{1}(M N-1)^{2}} \\
& \cdot\left[k_{1} k_{2}(M N-1)+k_{2}(W-1)(M-1)\right. \\
& \left.+k_{1}(W-1)(N-1)+(W-1)(N-1)(M-1)\right] \\
& \cdot\left[k_{1}(W-1)(N-1)+(W-1)(N-1)(M-1)\right]
\end{aligned}
$$

and

$$
\begin{aligned}
\int_{0}^{\infty} G_{1}(f) G_{3}(f) d f \\
=\frac{P_{\mathrm{sr}}^{2}}{\left(k_{1}-1\right)^{2}\left(k_{2}-1\right) k_{2}^{2} \Delta f^{2}} \\
\quad \cdot \int_{0}^{\infty}\left[\sum_{w_{1}=1}^{W} \sum_{i_{1}=0}^{M-1} \sum_{j_{1}=0}^{N-1} a_{i_{1} j_{1}}^{(1)} a_{i_{1} j_{1}}\left(w_{1}\right) F\left(f, i_{1}\right)\right]
\end{aligned}
$$

$$
\begin{aligned}
& \cdot\left[\sum_{w_{2}=1}^{W} \sum_{i_{2}=0}^{M-1} \sum_{j_{2}=0}^{N-1} a_{i_{2} j_{2}}^{(3)} a_{i_{2} j_{2}}\left(w_{2}\right) F\left(f, i_{2}\right)\right] d f \\
= & \frac{P_{\mathrm{sr}}^{2}}{\left(k_{1}-1\right)^{2}\left(k_{2}-1\right) k_{2}^{2} \Delta f^{2}} \\
& \cdot \frac{\Delta f}{M} \sum_{i=0}^{M-1}\left\{\left[\sum_{w_{1}=1}^{W} \sum_{j_{1}=0}^{N-1} a_{i_{1} j_{1}}^{(1)} a_{i_{1} j_{1}}\left(w_{1}\right)\right]\right. \\
= & \left.\left.\frac{P_{\mathrm{sr}}^{2}}{\left(k_{1}-1\right)^{2}\left(k_{2}-1\right) k_{2}^{2} M \Delta f} \sum_{w_{2}=0}^{N-1} a_{i_{2} j_{2}}^{(3)} a_{i_{2} j_{2}}\left(w_{2}\right)\right]\right\} \\
& \cdot\left[\frac{\left.k_{2}-1\right)^{2}}{\left(k_{1}-1\right)} \frac{(W-1)(M-1)}{(M N-1)}\right. \\
& \left.+\frac{1}{\left(k_{1}-1\right)} \frac{(W-1)(M-1)(N-1)}{(M N-1)}\right] \\
& \cdot\left[\frac{\left(k_{2}-1\right)}{\left(k_{1}-1\right)} \frac{(W-1)(M-1)(N-1)}{(M N-1)}\right] \\
& \frac{P_{\mathrm{sr}}^{2}}{M \Delta f k_{2}^{2}\left(k_{1}-1\right)^{2}(M N-1)^{2}} \\
& \cdot\left[k_{2}(W-1)(M-1)+(W-1)(N-1)(M-1)\right] \\
& \cdot[(W-1)(N-1)(M-1)] .
\end{aligned}
$$

Therefore, by substituting (29)-(35) into (28), and under the condition that all of the users send " 1 ," PIIN can be expressed as

$\left\langle i_{\text {PIIN }}^{2}\right\rangle$

$$
\begin{aligned}
= & \frac{R^{2} B_{\mathrm{r}} P_{\mathrm{sr}}^{2}}{M \Delta f k_{2}^{2}(M N-1)^{2}} \\
& \cdot\left\{\frac{1}{k_{1}}\left[\Lambda_{1}+\Lambda_{2}+\Lambda_{3}+\Lambda_{4}\right]^{2}+\frac{1}{\left(k_{1}-1\right)^{2}}\left[\Lambda_{2}+\Lambda_{4}\right]^{2}\right. \\
& +\frac{1}{k_{1}}\left[\Lambda_{3}+\Lambda_{4}\right]^{2}+\frac{1}{\left(k_{1}-1\right)^{2}}\left[\Lambda_{4}\right]^{2} \\
& -\frac{1}{k_{1}}\left[\Lambda_{1}+\Lambda_{2}+\Lambda_{3}+\Lambda_{4}\right]\left[\Lambda_{3}+\Lambda_{4}\right] \\
& \left.-\frac{1}{\left(k_{1}-1\right)^{2}}\left[\Lambda_{2}+\Lambda_{4}\right]\left[\Lambda_{4}\right]\right\} \\
= & \frac{R^{2} B_{\mathrm{r}} P_{\mathrm{sr}}^{2}}{M \Delta f k_{2}^{2}(M N-1)^{2}}\left[\frac{1}{k_{1}}\left(\Lambda_{1}+\Lambda_{2}\right)^{2}+\frac{\Lambda_{2}^{2}}{\left(k_{1}-1\right)^{2}}\right]
\end{aligned}
$$

where

$$
\begin{aligned}
& \Lambda_{1}=k_{1} k_{2}(M N-1) \\
& \Lambda_{2}=k_{2}(W-1)(M-1) \\
& \Lambda_{3}=k_{1}(W-1)(N-1)
\end{aligned}
$$


and

$$
\Lambda_{4}=(W-1)(M-1)(N-1) .
$$

However, since " 1 " and " 0 " are sent with equal probability for every user, (36) needs to be further amended and, consequently, we have

$$
\begin{aligned}
&\left\langle i_{\mathrm{PIIN}}^{2}\right\rangle=\frac{1}{2} \frac{R^{2} B_{\mathrm{r}} P_{\mathrm{sr}}^{2}}{M \Delta f k_{2}^{2}(M N-1)^{2}} \\
& \cdot\left[\frac{1}{k_{1}}\left(\Lambda_{1}+\Lambda_{2}\right)^{2}+\frac{\Lambda_{2}^{2}}{\left(k_{1}-1\right)^{2}}\right] .
\end{aligned}
$$

Since the shot noises come from PDs $0-3$ of the receiver are mutually independent, the power of the shot noise can be expressed as

$$
\left\langle i_{\text {shot }}^{2}\right\rangle=2 e B_{\mathrm{r}}\left(I_{0}+I_{1}+I_{2}+I_{3}\right) .
$$

After substituting (22)-(25) to (42), under the condition that all of the users send " 1 ," we have

$$
\begin{aligned}
&\left\langle i_{\text {shot }}^{2}\right\rangle=2 e B_{\mathrm{r}} \frac{P_{\mathrm{sr}} R}{k_{2} M} \\
& \cdot\left[k_{1} k_{2}+2 k_{1} \frac{(W-1)(N-1)}{(M N-1)}\right. \\
&+2 k_{2} \frac{(W-1)(M-1)}{(M N-1)} \\
&\left.+4 \frac{(W-1)(M-1)(N-1)}{(M N-1)}\right] .
\end{aligned}
$$

Again, since " 1 " and " 0 " are sent with equal probability for every user, (43) needs to be further amended as

$$
\begin{aligned}
\left\langle i_{\text {shot }}^{2}\right\rangle= & e B_{\mathrm{r}} \frac{P_{\mathrm{sr}} R}{k_{2} M} \\
& \cdot\left[k_{1} k_{2}+2 k_{1} \frac{(W-1)(N-1)}{(M N-1)}\right. \\
& +2 k_{2} \frac{(W-1)(M-1)}{(M N-1)} \\
& \left.+4 \frac{(W-1)(M-1)(N-1)}{(M N-1)}\right] .
\end{aligned}
$$

Finally, using the results of (26), (41), and (44), we obtain the signal-to-noise ratio (SNR) at the receiver as

$$
\mathrm{SNR}=\frac{I_{\mathrm{r}}^{2}}{\left\langle i_{\text {PIIN }}^{2}\right\rangle+\left\langle i_{\text {shot }}^{2}\right\rangle+\left\langle i_{\text {thermal }}^{2}\right\rangle}
$$

where

$$
\left\langle i_{\text {thermal }}^{2}\right\rangle=\frac{4 K_{b} T_{n} B_{\mathrm{r}}}{R_{L}} .
$$

TABLE V

Parameters Used in the Numerical Calculation

\begin{tabular}{c|c}
\hline PD quantum efficiency & $\eta=0.6$ \\
\hline Spectral width of broadband light source & $\begin{array}{c}\Delta \lambda=30 \mathrm{~nm} \\
\text { (i.e. } \Delta f=3.75 \mathrm{THz})\end{array}$ \\
\hline Wavelength location & $1.55 \mu \mathrm{m}$ \\
\hline Electrical bandwidth & $B=320 \mathrm{MHz}$ \\
\hline Data transmission rate & $622 \mathrm{Mbps}$ \\
\hline Receiver noise temperature & $T_{n}=300 \mathrm{~K}$ \\
\hline Receiver load resistor & $R_{L}=1030 \Omega$ \\
\hline
\end{tabular}

Furthermore, the BER can then be estimated from SNR as [11]

$$
\mathrm{BER}=\frac{\operatorname{erfc}\left(\sqrt{\frac{\mathrm{SNR}}{8}}\right)}{2}
$$

where

$$
\operatorname{erfc}(x)=\frac{2}{\sqrt{\pi}} \int_{x}^{\infty} \exp \left(-z^{2}\right) d z
$$

\section{Numerical AND Simulation Results}

The system parameters used to obtain the numerical results are listed in Table $\mathrm{V}$. We assume that the quantum efficiency of the photodiodes of the receivers is $\eta=0.6$. The receiver noise temperature and load resistance are $T_{n}=300 \mathrm{~K}$ and $R_{L}=1030 \Omega$. The spectrum of the broadband light source is centered at $1.55 \mu \mathrm{m}$ with spectral width $\Delta \lambda=30 \mathrm{~nm}$. The data transmission rate is $622 \mathrm{Mb} / \mathrm{s}$. For convenience of comparison to the systems proposed in [11] and [12], the electrical bandwidth of receivers is set as $320 \mathrm{MHz}$ to obtain the numerical results shown in Figs. 4-6.

Fig. 4 shows the number of simultaneous users of our proposed system using 2-D perfect difference codes versus the BER with similar code lengths when the effective source power is fixed at $-10 \mathrm{dBm}$ [the effective source power is $\alpha P_{s}$, in which $\alpha$ is caused by loss due to transmission and star coupler and is equal to $1 /(M N)$ when transmission loss is ignored]. For comparison, the numbers of simultaneous users for systems using MQC codes and M-matrices codes are also shown [11], [12].

As shown in Fig. 4, we use the codewords with $M=21$, $N=7$ and $M=57, N=3$. Their code lengths are 147 and 171, respectively. Thus, their code sizes are also 147 and 171, respectively. Since each code sequence can be assigned to a different subscriber, the maximum number of the subscribers in our proposed system can reach 147 and 171 by using these two codewords. For comparison, we consider the MQC code with $p=13$, whose code length and code size are 182 and 169 , respectively, and the M-matrices codes with $M=63$ and $N=3(N=3$ is the optimal case for the system using $\mathrm{M}$ matrices codes [12]), whose code length is 189 , which is the same as the code size thereof.

Fig. 4 shows that the proposed system can accommodate much more simultaneous users than the system using MQC codes or M-matrices codes. It means that with a similar code length, the proposed system has better performance than the other two systems. In addition, Fig. 4 also shows that the 


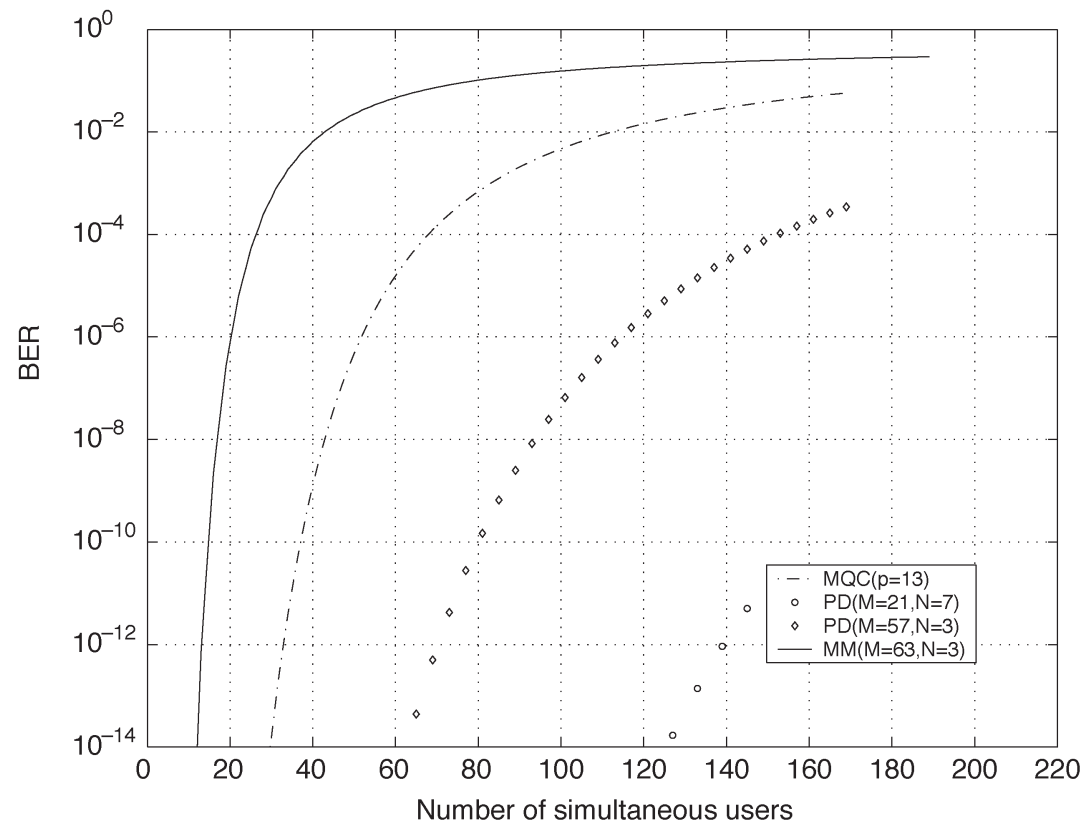

Fig. 4. Number of simultaneous users versus BER with similar code lengths and effective source power fixed at $-10 \mathrm{dBm}$.

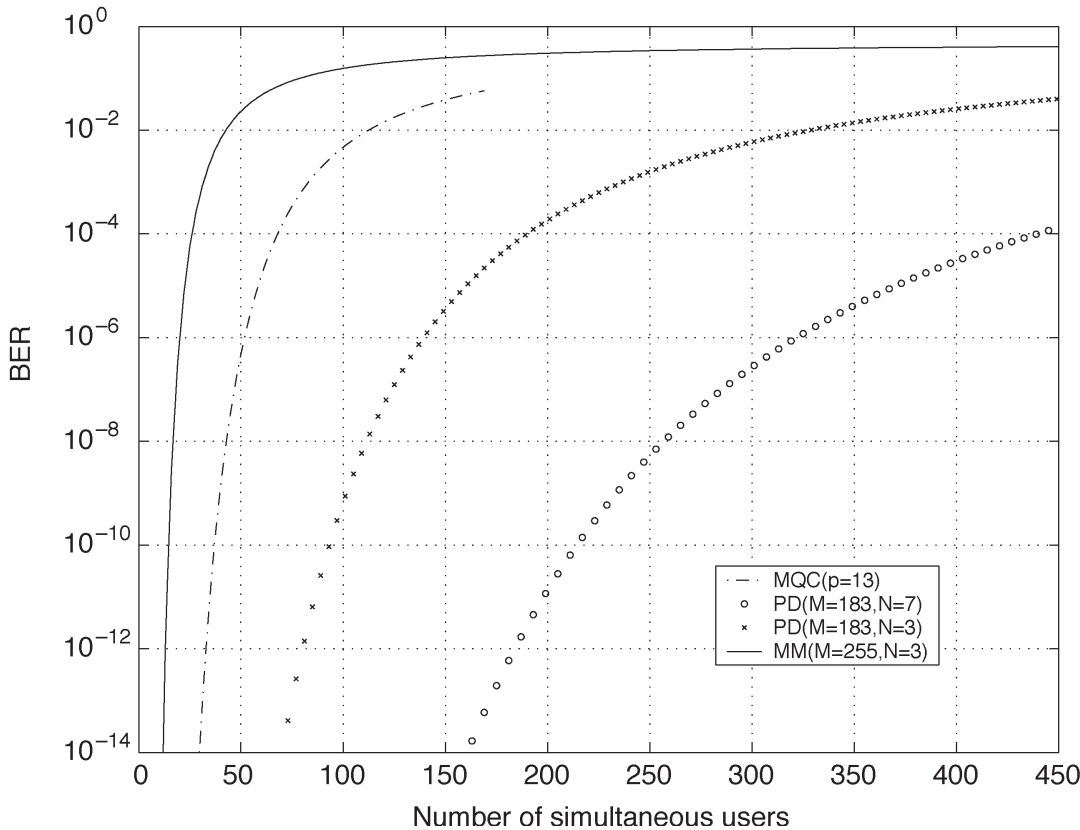

Fig. 5. Number of simultaneous users versus BER with similar spectral code lengths and effective source power fixed at $-10 \mathrm{dBm}$.

proposed system with $M=21$ and $N=7$ provides better performance than that with $M=57$ and $N=3$ does. Since $N$ represents the number of fibers employed for each user, this numerical result means that the performance of the proposed system can be improved by increasing the number of employed fibers under the condition of using similar code lengths.

Fig. 5 shows the number of simultaneous users of our proposed system versus the BER with similar spectral code lengths when the effective source power is fixed at $-10 \mathrm{dBm}$. For comparison, the numbers of simultaneous users for systems using MQC codes and M-matrices codes are also shown. Here, we use the codewords with $M=183$ and $N=3$ or 7 . Thus, their code sizes are 549 and 1281. For comparison, we consider the MQC code with $p=13$ and the M-matrices codes with $M=255$ and $N=3$, whose code length is 765 the same as the code size thereof.

Likewise, Fig. 5 also shows that the proposed system can accommodate more simultaneous users than the system using MQC codes or M-matrices codes. It means that with a similar spectral code length, the proposed system has better performance than the other two systems. In addition, Fig. 5 also shows that the proposed system with $M=183$ and $N=7$ provides better performance than that with $M=183$ and $N=3$. This result is reasonable, because the former case uses more fibers than the latter one. 


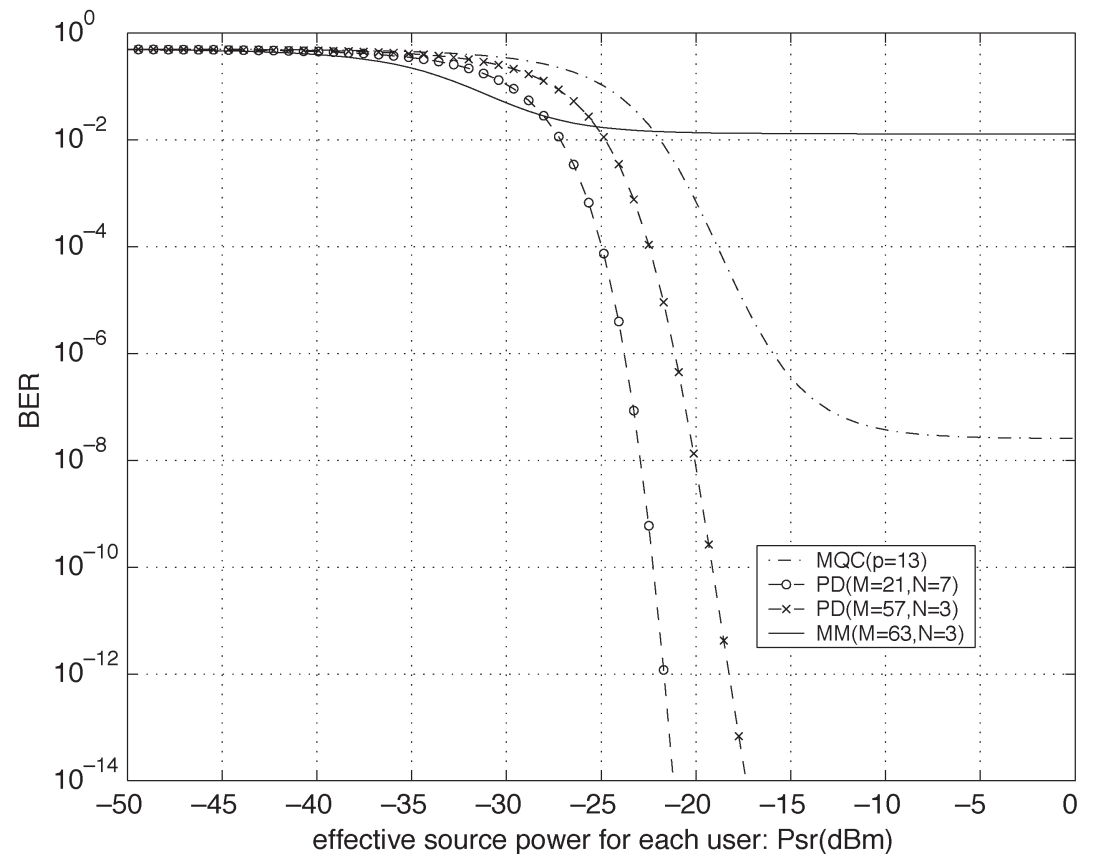

Fig. 6. Effective source power versus BER with similar code lengths when the number of simultaneous users is about 45 and the data rate of each user is $622 \mathrm{Mb} / \mathrm{s}$.

From Fig. 5, it is obvious that the proposed system with $M=183$ and $N=3$ can accommodate almost 2.5 times the maximum number of simultaneous users that can be accommodated by the system using the MQC codes with $p=13$. Similarly, the proposed system with $M=183$ and $N=7$ can accommodate almost six times the maximum number of simultaneous users that can be accommodated by the system using the MQC codes with $p=13$. It means that the number of simultaneous users of the proposed system is almost linearly proportional to the code length of the spatial codes, i.e., $N$. This feature can be used in system design for considering the tradeoff between the increment of cost that is mainly caused by using additional fibers and star couplers and the number of simultaneous users that can be accommodated in the proposed system.

Furthermore, Fig. 6 shows the effective source power versus the BER with similar code lengths when the number of simultaneous users is 45 at a data rate $622 \mathrm{Mb} / \mathrm{s}$ for each user. This figure proves that, compared with the systems using M-matrices codes and MQC codes, the proposed system can meet the optical transmission requirements with lower effective source power when the number of simultaneous users is the same. Furthermore, Fig. 6 also shows that the necessary effective source power needed for the proposed system with $M=21$ and $N=7$ is less than that needed for the proposed system with $M=57$ and $N=3$. It means that the necessary effective source power can be smaller when the spatial code length is larger. It is so, because the power of interference from other users is reduced with the increase of the spatial code length.

In addition, Fig. 7 shows the data transmission rate of each user versus the BER with similar code lengths when the number of simultaneous users is 45 and the effective source power is $-10 \mathrm{dBm}$. It should be noted that, in order to meet the requirement of data reception for practical systems, the electrical bandwidth of receivers is set to be the data transmission rate multiplied by 0.7 . Fig. 7 proves that the proposed system can provide a higher data transmission rate for each user when a similar code length is used. Furthermore, Fig. 7 also shows that the available data transmission rate for the proposed system with $M=21$ and $N=7$ is larger than that for the proposed system with $M=57$ and $N=3$. It means that the available data transmission rate for each user can be increased when the spatial code length is larger.

Fig. 8 shows theoretical estimations and simulation results, which are acquired by using a software tool named "VPItransmissionMaker," of the effective source power versus the BER for the system using perfect difference codes with $M=3$ and $N=3$. In the simulation, we use light-emitting diodes (LEDs) as the broadband sources and FBGs to implement the encoders and decoders.

Since $M=3$, the code weight of the used spectral codes is $k_{1}=2$. Hence, each set of the FBGs in the encoders and decoders is made to reflect two spectral components back and let the remainder pass through. In addition, each of the spectral components is set to have a bandwidth with $150 \mathrm{GHz}$. Thus, the effective bandwidth of the source is $\Delta f=M \times 150 \mathrm{GHz}$, i.e., $0.45 \mathrm{THz}$. Moreover, the data rate is $622 \mathrm{Mb} / \mathrm{s}$ and the electrical bandwidth of the receivers is $320 \mathrm{MHz}$. Each receiver has four $\mathrm{p}$-i-n diodes with quantum efficiency $\eta=0.6$.

Fig. 8 shows the theoretical estimations and the simulation results for $W=1$ and $W=4$, where $W$ represents the number of simultaneous users. As shown in the figure, the theoretical estimations are quite close to the simulation results. The difference between the theoretical estimation and simulation result is smaller than $2 \mathrm{~dB}$ at $\mathrm{BER}=10^{-9}$ for $W=1$ and about $3 \mathrm{~dB}$ for $W=4$. The differences between the theoretical estimations and the simulation results are caused by various nonideal properties and power loss of the practical optical components. For example, the FBGs are unable to provide 


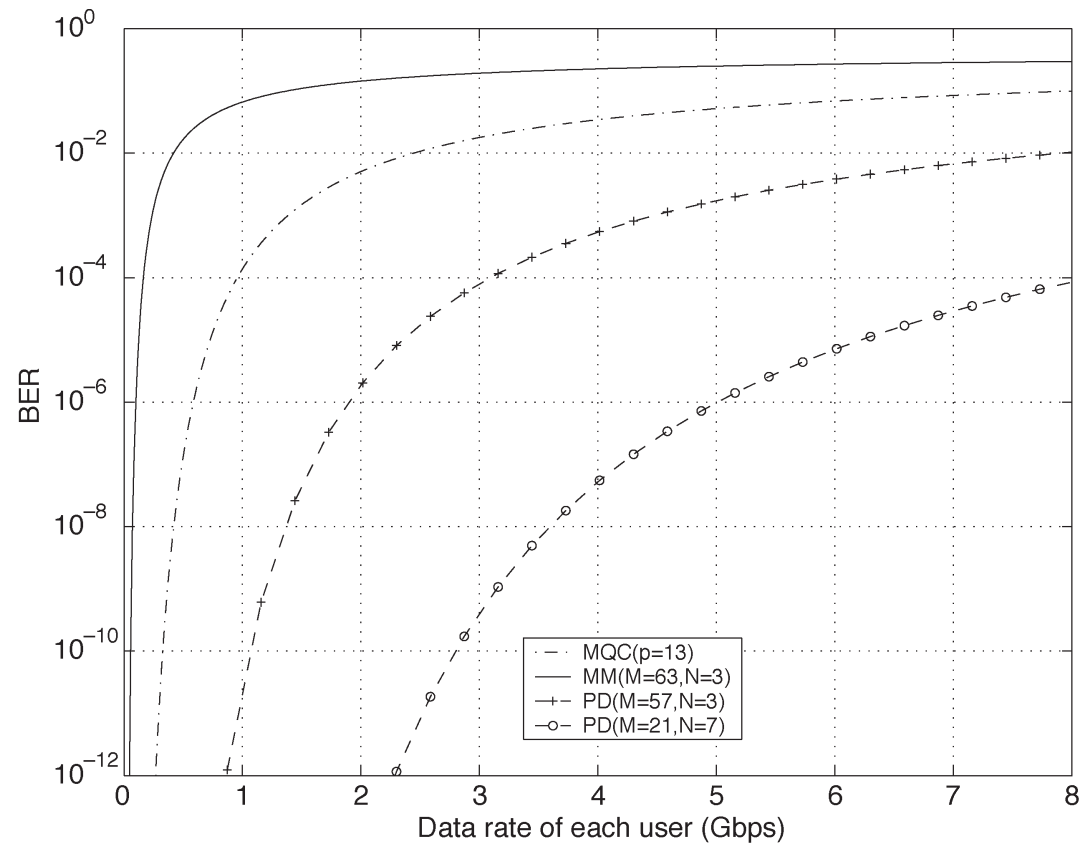

Fig. 7. Data transmission rate versus BER with similar code lengths when the number of simultaneous users is about 45 and the effective source power is $-10 \mathrm{dBm}$

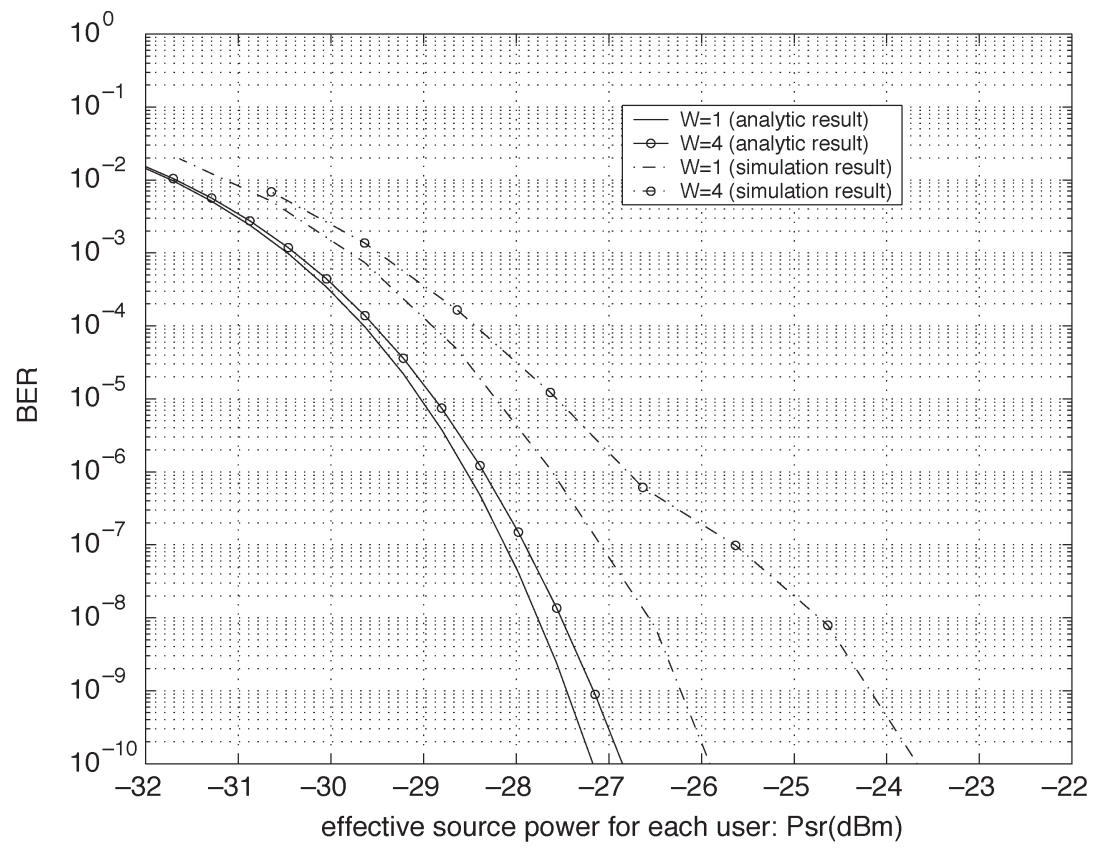

Fig. 8. Theoretical estimations and simulation results of the effective source power versus BER for the system using 2-D perfect difference codes with $M=3$ and $N=3$.

the ideal filtering function and the spectrum of the LEDs is not flat.

\section{DISCUSSIONS}

According to the description and analysis mentioned above, several critical parameters, i.e., system complexity, spectral code length, spatial code length, effective source power, and data transmission rate, in the proposed system, and their relations need to be further discussed. Compared with the systems using M-matrices and MQC codes, the proposed system has a structure with middle complexity. In fact, the complexity of the network structure for the proposed system is almost the same as that for the system using M-matrices codes and nearly $N$ times greater than that for the system using MQC codes, where $N$ is the spatial code length that is used.

As for the transmitter and receiver of the proposed system, their complexity is much smaller than that of the system using M-matrices codes and almost twice greater than that of the system using MQC codes. Because the code weight of the spectral codes of the 2-D perfect difference codes is much smaller than that of the M-matrices codes with a similar code 
length, the number of fiber gratings, which is used to obtain the spectral components corresponding to the spectral codes, needed for the proposed system is also much fewer than that for the system using M-matrices codes. Hence, the complexity of the transmitter and receiver of the proposed system is much smaller than that of the system using M-matrices codes. Furthermore, for decoding, the receiver of the proposed system has two balanced detectors, each of which has a similar structure as that of the receiver of the system using MQC codes. Hence, the complexity of the proposed system is almost twice greater than that of the system using MQC codes.

Clearly, the complexities of the network structure and the transmitter/receiver structure of the proposed system are related to the spatial and spectral code lengths used. The complexity of the network structure is linearly proportional to the spatial code length, which is proportional to the numbers of fibers and star couplers. The complexity of the transmitter/receiver structure is linearly increased with the code weight of the spectral codes, because the number of the fiber gratings used in the transmitters and receivers linearly proportional to the code weight of the spectral codes. Because the power of interference from other users is reduced with the increase of the spatial code length, the necessary effective source power needed for the proposed system can be lower when the spatial code length is larger. At the same time, the available data transmission rate for each user can also be increased.

Compared with the systems using M-matrices and MQC codes, the proposed system has a structure with middle complexity. The complexity of the proposed system is increased when the spectral or spatial code length is increased. However, the increase of the spectral or spatial code length makes the proposed system able to accommodate more simultaneous users. In addition, the increase of the spatial code length lowers the necessary effect source power and increases available data transmission rate for each user. Therefore, the system designer can make a tradeoff between system complexity and system performance.

\section{CONCLUSION}

In this paper, we present a family of new codes named twodimensional (2-D) perfect difference codes to lower the cross correlation of any two different codewords so as to suppress phase-induced intensity noise (PIIN). The 2-D perfect difference codes are constructed in view of one-dimensional (1-D) perfect difference codes. Every codeword of the 2-D perfect difference codes is composed of a spectral code sequence and a spatial code sequence. Since both of the spectral and spatial code sequences are selected from the 1-D perfect difference codes, the 2-D perfect difference codes have a multiuser interference (MUI) cancellation property. By using this property, the interference from other users can be completely removed.

Since the in-phase cross correlations of the spectral and spatial code sequences are exactly equal to 1 , the cross correlation value of the 2-D perfect difference codes is much smaller than that of the M-matrices codes. Hence, using the 2-D perfect difference codes can greatly reduce the PIIN. Furthermore, in comparison with the system using the MQC codes, the pro- posed system designed according to the 2-D perfect difference codes can effectively reduce the power of beating signals sent from other users. In accordance with the numerical results, the system performance can be improved by increasing the number of employed fibers, i.e., increasing the spatial code length $N$, under the condition of using similar code lengths. Moreover, under the same condition, the maximum number of simultaneous users is almost linearly proportional to the code length of the used spatial codes.

Furthermore, if the code lengths are similar, the necessary effective source power of the proposed system is lower and the available data transmission rate is higher than that of other systems. However, since the complexity of the proposed system is increased with the spatial and spectral code lengths of the 2-D perfect difference codes, there is a tradeoff between the complexity and system performance. In addition, the theoretical estimations of the proposed system are verified by the simulation results.

\section{REFERENCES}

[1] P. R. Prucnal, M. A. Santoro, and T. R. Fan, "Spread spectrum fiberoptic local area network using optical processing," J. Lightw. Technol., vol. LT-4, no. 5, pp. 547-554, May 1986.

[2] W. C. Kwong, P. A. Perrier, and P. R. Prucnal, "Performance comparison of asynchronous and synchronous code-division multiple-access techniques for fiber-optic local area networks," IEEE Trans. Commun., vol. 39, no. 11 , pp. 1625-1634, Nov. 1991.

[3] C.-S. Weng and J. Wu, "Perfect difference codes for synchronous fiberoptic CDMA communication systems," J. Lightw. Technol., vol. 19, no. 2, pp. 186-194, Feb. 2001.

[4] J. A. Salehi, "Code division multiple-access techniques in optical fiber networks-Part I: Fundamental principles," IEEE Trans. Commun., vol. 37, no. 8, pp. 824-833, Aug. 1989.

[5] F. R. K. Chung, J. A. Salehi, and V. K. Wei, "Optical orthogonal codes: Design, analysis, and applications," IEEE Trans. Inf. Theory, vol. 35, no. 3, pp. 595-604, May 1989.

[6] H. Fathallah, L. A. Rusch, and S. LaRochelle, "Passive optical fast frequency-hop CDMA communications system," J. Lightw. Technol., vol. 17, no. 3, pp. 397-405, Mar. 1999.

[7] E. Inaty, H. M. H. Shalaby, and P. Fortier, "On the cutoff rates of a multiclass OFFH-CDMA system," IEEE Trans. Commun., vol. 53, no. 2, pp. 323-334, Feb. 2005.

[8] M. Kavehrad and D. Zaccarin, "Optical code-division-multiplexed systems based on spectral encoding of noncoherent sources," J. Lightw. Technol., vol. 13, no. 3, pp. 534-545, Mar. 1995.

[9] E. D. J. Smith, R. J. Blaikie, and D. P. Taylor, "Performance enhancement of spectral amplitude-coding optical CDMA using pulse-position modulation," IEEE Trans. Commun., vol. 46, no. 3, pp. 1176-1185, Sep. 1998.

[10] X. Zhou, H. H. M. Shalaby, C. Lu, and T. Cheng, "Code for spectral amplitude coding optical CDMA systems," Electron. Lett., vol. 36, no. 8, pp. 728-729, Apr. 13, 2000.

[11] Z. Wei, H. M. H. Shalaby, and H. Ghafouri-Shiraz, "Modified quadratic congruence codes for fiber Bragg-grating-based spectral-amplitudecoding optical CDMA systems," J. Lightw. Technol., vol. 19, no. 9, pp. 1274-1281, Sep. 2001.

[12] C.-C. Yang and J.-F. Huang, "Two-dimensional M-matrices coding in spatial/frequency optical CDMA networks," IEEE Photon. Technol. Lett., vol. 15 , no. 1, pp. 168-170, Jan. 2003.

[13] J. Singer, "A theorem in finite projective geometry and some applications to number theory," Trans. Amer. Math. Soc., vol. 43, no. 3, pp. 377-385, 1938.

[14] E. D. J. Smith, R. J. Blaikie, and D. P. Taylor, "Performance enhancement of spectral amplitude-coding optical CDMA using pulse-position modulation," IEEE Trans. Commun., vol. 46, no. 3, pp. 1176-1185, Sep. 1998.

[15] J. W. Goodman, Statistical Optics. New York: Wiley, 1985.

[16] A. Meijerink, G. H. L. M. Heideman, and W. C. van Etten, "A generalization of a coherence multiplexing system," in Proc. Symp. Communication and Vehicular Technology, SCVT-200, Leuven, Belgium, Oct. 19, 2000, pp. 6-13. 


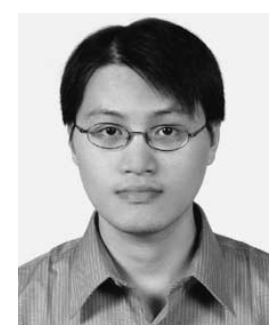

Cheing-Hong Lin was born in Taipei, Taiwan, R.O.C., in 1975. He received the B.S. degree in communication engineering from the National Chiao Tung University, Hsinchu, Taiwan, in 1997, and the M.S. and Ph.D. degrees in communication engineering from the National Taiwan University, Taipei, in 1999 and 2005, respectively.

Currently, he is with ZyXEL Communications Corporation, Taiwan, as a Research Engineer. His research interests include lightwave and wireless communication systems and computer networks.

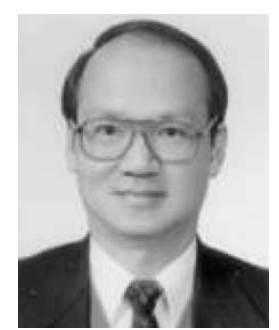

Jingshown Wu (S'73-M'78-SM'99-F'05) received the B.S. and M.S. degrees in electrical engineering from the National Taiwan University, Taipei, Taiwan, R.O.C., and the Ph.D. degree from Cornell University, Ithaca, NY, in 1970, 1972, and 1978, respectively.

He joined Bell Laboratories in 1978, where he worked on digital network standards and performance, and optical fiber communication systems. In 1984, he joined the Department of Electrical Engineering of the National Taiwan University as a Professor and was the Chairman from 1987 to 1989. He was also the Director of the Communication Research Center, College of Engineering of the university from 1992 to 1995. From 1995 to 1998, he was the Director of the Division of Engineering and Applied Science, National Science Council, Taiwan, R.O.C., on leave from the university. From 1999 and 2002, he was the Chairman of the Commission on Research and Development and the Director of the Center for Sponsor Programs of the National Taiwan University. His interests are optical fiber communications, computer communications, and communication systems. He has published more than 100 journal and conference papers and holds 11 patents.

Prof. Wu is a Life Member of the Chinese Institute of Engineers, the Optical Society of China, and the Institute of Chinese Electrical Engineers. He received the Distinguished Research Awards from National Science Council from 1991 to 1996 and the Outstanding Engineering Professor Award from the Chinese Institute of Engineers in 1996. He served as the Vice Chairman (1997-1998) and the Chairman (1998-2000) of IEEE Taipei Chapter.

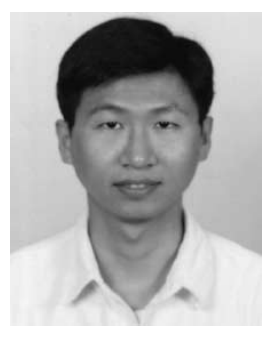

Chun-Liang Yang was born in I-Lan, Taiwan, R.O.C., in May 1971. He received the B.S., M.S., and $\mathrm{Ph} . \mathrm{D}$. degrees in electronic engineering from the National Taiwan University of Science and Technology (NTUST), Taipei, Taiwan, in 1996, 1998, and 2004, respectively.

$\mathrm{He}$ is currently an Assistant Professor at the Department of Electrical Engineering, Tamkang University (TKU), Taipei, Taiwan. His research interests include optical performance monitoring and optical networks. 